\title{
Correction to "Hepatitis C for Primary Care Physicians"
}

In the above-mentioned article, ${ }^{1}$ the sustained virological response (SVR) is defined as "no detectable hepatitis C viral RNA 24 months after treatment." The time frame should read " 24 weeks" and not months. The electronic version on the Journal of the American Board of Family Medicine website has been corrected. We apologize for the error, and we regret any confusion or inconvenience it may have caused.

doi: 10.3122/jabfm.2014.04.140144

\section{Reference}

1. Huffman MM, Mounsey AL. Hepatitis C for primary care physicians. J Am Board Fam Med 2014;27:284-91. 